\title{
ARTICLE
}

Cite this: DOI: 10.1039/x0xx00000x

Received 00th December 2013, Accepted 00th January 2013

DOI: $10.1039 / \times 0 \times x 00000 x$

www.rsc.org/

\section{All-conjugated cationic copolythiophene "rod- rod" block copolyelectrolytes: synthesis, optical properties and solvent-dependent assembly}

\author{
Amandine Thomas, ${ }^{\text {ab }}$ Judith E. Houston, ${ }^{\mathrm{c}}$ Niko Van den Brande, ${ }^{\mathrm{d}}$ Julien De \\ Winter, ${ }^{\mathrm{e}}$ Michèle Chevrier, ${ }^{\text {af }}$ Richard K. Heenan, ${ }^{\mathrm{g}}$ Ann E. Terry, ${ }^{\mathrm{g}}$ Sébastien \\ Richeter, ${ }^{a}$ Ahmad Mehdi, ${ }^{a}$ Bruno Van Mele, ${ }^{\mathrm{d}}$ Philippe Dubois, ${ }^{\mathrm{e}}$ Roberto \\ Lazzaroni, ${ }^{\mathrm{b}}$ Pascal Gerbaux, ${ }^{\mathrm{e}}$ Rachel C. Evans* ${ }^{* \mathrm{ch}}$ and Sébastien Clément*a
}

\begin{abstract}
Amphiphilic diblock copolythiophenes were synthesised by an efficient two-step synthetic strategy. The diblock copolyelectrolytes were obtained via the quasi-living Kumada catalysttransfer polycondensation followed by quaternisation of the bromohexyl side chains of one of the monomer constituents into $\mathrm{N}$-methylimidazolium, pyridinium, trimethylammonium or trimethylphosphonium units. The effects of the nature of the charged group on the thermal properties were investigated by Rapid Heat-Cool (RHC) calorimetry measurements. The solvent-driven assembly of these block copolyelectrolytes in chloroform $\left(\mathrm{CHCl}_{3}\right)$, water, methanol $(\mathrm{MeOH})$, water: $\mathrm{MeOH}$ mixtures and in subsequently prepared thin films was investigated using a combination of photoluminescence, scattering and microscopic techniques. The rigid rod-structure of the block copolyelectrolytes lead to the formation of core-shell cylindrical aggregates in solution, with features determined by the nature of the solvent. AFM studies revealed that the aggregates formed in solution can be transferred into thin films allowing for the reliable control of the self-organisation process and the resulting nanoscale architecture. Such copolymers are, therefore, promising candidates for application as the active layer in electronic devices such as organic solar cells.
\end{abstract}

\section{Introduction}

Conjugated polyelectrolytes (CPEs) are defined as polymers with an extended $\pi$-conjugated backbone and ionic pendant groups, which combine the organic semiconductor properties and charge-mediated characteristics of polyelectrolytes in a single functional material. ${ }^{1}$ As such, CPEs constitute a promising class of materials for application in optoelectronic devices, chemo- and biosensors, and biological imaging. ${ }^{2}$ However, since device performance depends on both the optoelectronic properties and nanoscale morphology of the polymer (which are intrinsically linked), understanding the parameters and physical processes influencing the polymer morphology and its interdependent optoelectronic properties is of crucial importance. ${ }^{3}$

Recently, self-assembly strategies have emerged as an elegant approach for the design and fabrication of reproducible nanoscale aggregates from conjugated polyelectrolyte building blocks. ${ }^{4}$ For example, co-assembly with oppositely charged species in solution (e.g. ionic surfactants, ${ }^{5}$ metal ions ${ }^{6}$ and complexes/clusters, ${ }^{7}$ DNA $^{8}$ ) is facilitated via electrostatic interactions with the charged side-chains, leading to the formation of extended networks exhibiting structural and morphological organisation across multiple length scales.

Block copolymers may also provide an alternative interesting route to control the self-assembly into nanostructured morphologies. ${ }^{9}$ However, the rodlike nature of most conjugated polymers complicates the self-assembly of corresponding block copolymers through an interplay of nanophase separation and bulk crystallisation. ${ }^{9 a}$ Nonetheless, self-organisation at the nanoscale into lamellar/fibrillar morphology can be obtained in all-conjugated rod-rod block copolymers. ${ }^{10}$ The self-assembly of such copolymers into stable nanostructures of different shape or morphology is dictated by both the chain stiffness and the solubility of the individual blocks. ${ }^{11}$ In this respect, the combination of covalently-bound ionic and neutral blocks can be used to introduce solubility gradients across the copolymer structure and subsequent selfassembly into organised nanostructures can be induced by solvent-mediation. ${ }^{12}$

Scherf et al. have in particular reported that all-conjugated cationic "rod-rod" block copolyelectrolytes containing two electronically-different conjugated blocks (namely, 
polyfluorene and polythiophene blocks) exhibit solventmediated self-assembly in mixtures of selective and nonselective solvents, such as water-methanol $(\mathrm{MeOH})$ and waterTHF, which provides a convenient method of modulating the photoluminescence properties via segregation of the two blocks. ${ }^{12}$ Control of the nanomorphology of the self-assembled aggregates (e.g. vesicles, rods, etc.) may be obtained through judicious selection of the concentration, solvent or molecular weight of the polymer. ${ }^{12}$ Similar changes in nanophase morphology were also observed in all-conjugated cationic diblock copolymers composed of two polyfluorene blocks (a neutral block bound to a cationic block) in protic or non-protic solvents (MeOH vs. THF). ${ }^{13}$

Maes et al. have recently reported a family of ionic polythiophene-based homopolymers and random copolymers functionalised with imidazolium-terminated side chains at different comonomer ratios. ${ }^{14}$ However, the analogous allconjugated cationic diblock polythiophene copolymers have been scarcely studied. This is somewhat surprising on two counts. Firstly, oligo- and polythiophenes are well-known to undergo temperature- and solvent-induced conformational changes which result in thermo- or solvato-chromatic responses in their optical properties (absorbance, fluorescence). ${ }^{15}$ Secondly, poly(3-hexylthiophene) (P3HT) is by far the most commonly used electron donor material in organic photovoltaics (OPV) ${ }^{16}$ and conjugated polyelectrolytes have also found application as interfacial layers in bulk heterojunction OPV devices. ${ }^{17}$ Significant efforts have been dedicated to the synthesis of sophisticated polythiophene derivatives, with the aim of improved morphological control and enhanced thermal stability. ${ }^{18}$ Amphiphillic diblock polythiophene copolymers are thus extremely attractive in this respect as they should afford concomitant tailoring of both the nanoscale morphology and the optical properties via solventinduced self-assembly.

Control of the molecular weight, and in particular, the requirement for a narrow molecular weight distribution, are also important parameters to be considered when designing new diblock copolymers for the purpose of self-assembly. ${ }^{9 a, f}$ Moreover, the molecular weight of ionic polythiophenes has been identified as a crucial parameter influencing the performance of OPV devices. ${ }^{18 a}$ In this respect, the Kumada Catalyst Transfer Polycondensation (KTCP) method, which enables chain-growth polymerisation and leads to the synthesis of conjugated polymers with relatively high molecular weight, narrow polydispersity and minimised termination side reactions, is a tool of choice to obtain ordered nanostructures. ${ }^{19}$ This method has been successfully applied to the synthesis of well-defined polyfluorenes, ${ }^{20}$ polypyrroles ${ }^{21}$ and polycarbazoles. $^{22}$ Moreover, the "living" chain growth mechanism of the KTCP reaction might afford straightforward access to regioregular block copolymers through a one-pot procedure. ${ }^{19 \mathrm{c}, 23}$

Herein, we report on the synthesis of amphiphilic "rod-rod" block copolyelectrolytes composed of two polythiophenes blocks (a neutral block bound to a cationic block) bearing either
$\mathrm{N}$-methylimidazolium, pyridinium, trimethylammonium or trimethylphosphonium groups prepared by using the KTCP method. The effects of the nature of the charged group on the optical and thermal properties are investigated. The aggregation properties of the CPEs in selective and non-selective solvent mixtures $\left(\mathrm{H}_{2} \mathrm{O}-\mathrm{MeOH}\right)$ are evaluated using a combination of dynamic light scattering (DLS), small-angle neutron scattering (SANS) and atomic force microscopy (AFM), with the goal of identifying key structure-property relationships in this novel class of materials.

\section{Experimental}

\section{Materials and characterisation methods}

All reactions were carried out under argon or nitrogen using standard high-vacuum and Schlenk techniques. 2-bromo-3hexyl-5-iodo-thiophene and 2,5-dibromo-3-bromohexylthiophene were prepared according to literature methods. ${ }^{10 a, 24}$ Dry THF was obtained by distilling over $\mathrm{CaH}_{2}$, then $\mathrm{Na}$ /benzophenone. Dry toluene was obtained by distilling over Na. Chemicals were obtained from Alfa-Aesar, Sigma-Aldrich and Acros and used as received. All NMR spectra were acquired with a Bruker Avance $300\left({ }^{1} \mathrm{H} 300.13 \mathrm{MHz},{ }^{13} \mathrm{C}\left\{{ }^{1} \mathrm{H}\right\}\right.$ 75.48 MHz and $\left.{ }^{31} \mathrm{P}\left\{{ }^{1} \mathrm{H}\right\} 121.49 \mathrm{MHz}\right)$ using the solvent as the chemical shift standard, except for ${ }^{31} \mathrm{P}\left\{{ }^{1} \mathrm{H}\right\}$ NMR, where the chemical shifts are relative to $85 \% \mathrm{H}_{3} \mathrm{PO}_{4}$ in $\mathrm{D}_{2} \mathrm{O}$. All chemical shifts and coupling constants are reported in ppm and $\mathrm{Hz}$, respectively. Elemental analyses were performed with a Thermofinigane Flash EA 1112 instrument and expressed in terms of the weight percent ratio of nitrogen to sulphur atoms. Number-averaged $\left(\mathrm{M}_{\mathrm{n}}\right)$, weight-averaged $\left(\mathrm{M}_{\mathrm{w}}\right)$ molecular weights and the molecular weight distribution (PDI) of P3HTb-P3HTBr were measured using size exclusion chromatography (SEC) on a Polymer Laboratories liquid chromatograph equipped with a PL-DG802 degasser, an isocratic HPLC pump LC 1120 (flow rate $=1 \mathrm{~mL} \mathrm{~min}^{-1}$ ), a Marathon autosampler (loop volume $=200 \mu \mathrm{L}$, solution conc. $=$ $\left.1 \mathrm{mg} \cdot \mathrm{mL}^{-1}\right)$, a PL-DRI refractive index detector and three columns: a PL gel $10 \mu \mathrm{m}$ guard column and two PL gel MixedB $10 \mu \mathrm{m}$ columns (linear columns for separation of MWPS ranging from 500 to $10^{6}$ daltons). The eluent used was THF at a flow rate of $1 \mathrm{~mL} \mathrm{~min}-1$ at $40^{\circ} \mathrm{C}$. Polystyrene standards were used to calibrate the SEC. Matrix-assisted laser desorption/ionisation time-of-flight (MALDI-ToF) mass spectra were recorded using a Water QToF Premier mass equipped with a nitrogen laser, operating at $337 \mathrm{~nm}$ with a maximum output of $500 \mathrm{~J} \mathrm{~m}^{-2}$ delivered to the sample in $4 \mathrm{~ns}$ pulses at 20 $\mathrm{Hz}$ repeating rate. Mass analyses were performed in reflection mode at a resolution of about 10,000. All samples were analyzed using (DCTB) trans-2-[3-(4-tertbutylphenyl)-2methylprop-2-enylidene] malononitrile. This matrix was prepared as a $40 \mathrm{mg} \mathrm{mL}{ }^{-1}$ solution in $\mathrm{CHCl}_{3} .{ }^{25}$ The matrix solution $(1 \mu \mathrm{L})$ was applied to a stainless steel target and air dried. Polymer samples were dissolved in $\mathrm{CHCl}_{3}$ to obtain $1 \mathrm{mg}$ $\mathrm{mL}^{-1}$ solutions. $1 \mu \mathrm{L}$ aliquots of these solutions were applied 
onto the target area already bearing the matrix crystals, and air dried. For the recording of the single-stage MS spectra, the quadrupole (rf-only mode) was set to pass ions from m/z 500 to $\mathrm{m} / \mathrm{z} 15,000$, and all ions were transmitted into the pusher region of the time-of-flight analyser where they were mass analyzed with $1 \mathrm{~s}$ integration time. Rapid Heat-Cool Calorimetry (RHC) experiments were performed on a prototype RHC from TA Instruments, equipped with liquid nitrogen cooling and specifically designed for operation at high scanning rates. ${ }^{26,27}$ RHC measurements were performed at $500 \mathrm{~K} \mathrm{~min}^{-1}$ in aluminium non-hermetic crucibles, using neon $\left(6 \mathrm{~mL} \mathrm{~min}^{-1}\right)$ as a purge gas. For comparison with block copolymers, pure CPE homopolymers were synthesized from a P3HTBr precursor $\left(\mathrm{M}_{\mathrm{n}}=14900, \mathrm{M}_{\mathrm{w}}=20700, \mathrm{PDI}=1.39\right)$ following a recently reported procedure. ${ }^{8} \mathrm{UV} / \mathrm{Vis}$ absorption and fluorescence spectra were recorded at room temperature on a Shimadzu UV2401 PC UV/Vis scanning spectrometer and a Fluorolog-3 (Horiba Jobin Yvon) spectrophotometer, respectively. Emission and excitation spectra were corrected for the wavelength response of the system using correction factors supplied by the manufacturer. Fluorescence quantum yields $\left(\Phi_{\mathrm{F}}\right)$ were measured using tetraphenylporphyrin (TPP) in toluene as a standard $\left(\Phi_{\mathrm{F}}=0.11\right) .{ }^{28}$ Dynamic light scattering measurements were performed using a Zetasizer Nano series nano-ZS (Malvern Instruments, U.K.) in the back-scattering configuration $\left(175^{\circ}\right)$. Cumulant analysis of the correlation function was used to determine the mean hydrodynamic diameter (Z-average) and the polydispersity of each sample. ${ }^{29}$ AFM measurements were performed using an Asylum Research MFP-3D ${ }^{\mathrm{TM}}$ instrument mounted on an anti-vibration plinth, in the tapping mode at room temperature under ambient conditions. The silicon cantilevers used were $125 \mu \mathrm{m}$ in length and had a resonance frequency of $\sim 150 \mathrm{kHz}$. All raw AFM images were visualised and analysed using the Gwyddion 2.31 software. SANS was carried out on the Sans2d small-angle diffractometer at the ISIS Pulsed Neutron Source (STFC Rutherford Appleton Laboratory, Didcot, U.K.). ${ }^{30}$ A simultaneous $q$-range of $\sim 0.004-0.6 \AA^{-1}$ was achieved utilising an incident wavelength range of $1.75-16.5 \AA$ separated by time-of-flight and employing an instrument set up of $\mathrm{L}_{1}=\mathrm{L}_{2}=$ $4 \mathrm{~m}$, with the $1 \mathrm{~m}^{2}$ detector offset vertically $150 \mathrm{~mm}$ and sideways $150 \mathrm{~mm} . q=(4 \pi / \lambda) \sin (\theta / 2)$ where $\lambda$ is the wavelength and $\theta$ the scattering angle. Samples were prepared in deuterated solvents, to provide good neutron scattering contrast. Each raw scattering data set was corrected for the detector efficiencies, sample transmission and background scattering and converted to scattering cross-section data $(\partial \Sigma / \partial \Omega$ vs. q) using the instrument-specific software. ${ }^{31}$ These data were placed on an absolute scale $\left(\mathrm{cm}^{-1}\right)$ using the scattering from a standard sample (a solid blend of hydrogenated and perdeuterated polystyrene) in accordance with established procedures. ${ }^{32}$ The scattering functions were fit using non-linear least-squares analysis to a Core-Shell-Cylinder model using the SasView programme. ${ }^{33}$ Full details of the models and the fitting procedure can be found in the Supporting Information.

\section{Synthesis}

Poly[(3-hexylthiophene)-2,5-diyl]-block-poly[3-(6bromohexyl)-thiophene-2,5-diyl] block copolymer (P3HT- $b$ P3HTBr). Two round-bottomed flasks $(100 \mathrm{~mL})$ were dried by heating under reduced pressure and cooled to room temperature. 2-Bromo-3-hexyl-5-iodothiophene (1.22 g, 3.3 $\mathrm{mmol})$ was dried by three successive azeotropic distillations with toluene, and then dry THF $(15 \mathrm{~mL})$ was added. A 2 mol.L1 solution of $i$-PrMgCl in THF $(1.65 \mathrm{~mL})$ was added via a syringe, and the mixture was stirred at $0^{\circ} \mathrm{C}$ for $30 \mathrm{~min}$ (solution A). Solution A was added in one portion to $\mathrm{Ni}(\mathrm{dppp}) \mathrm{Cl}_{2}$ catalyst $(59 \mathrm{mg})$ in THF $(10 \mathrm{~mL})$. In the other flask, $2.7 \mathrm{mmol}$ of 2,5-dibromo-3-bromohexylthiophene $(1.09 \mathrm{~g})$, previously dried by azeotropic distillation, was reacted with $i$-PrMgCl $(1.35 \mathrm{~mL})$ in the same manner (solution B). After stirring the reaction mixture for $3 \mathrm{~h}$, solution $\mathrm{B}$ was added to the previous mixture via a syringe, and the resulting solution was stirred overnight. The reaction was quenched quickly by pouring $\mathrm{HCl}$ aq $(5 \mathrm{M})$ into the solution and stirring for $0.5 \mathrm{~h}$. Then, the mixture was precipitated in cold $\mathrm{MeOH}$ and filtered. The product was washed well with $\mathrm{MeOH}$ and hexane to afford a red solid. P3HT-b-P3HTBr. Yield: 0.99 g (82\%). ${ }^{1} \mathrm{H}$ NMR $\left(\mathrm{CDCl}_{3}\right): \delta=0.90\left(\mathrm{t}, 3 \mathrm{H}, \mathrm{CH}_{3},{ }^{3} J_{\mathrm{H}-\mathrm{H}}=6.8 \mathrm{~Hz}\right), 1.62(\mathrm{~m}, 16 \mathrm{H}$, $\left.\mathrm{CH}_{2}\right), 2.80\left(\mathrm{t}, 4 \mathrm{H}, \mathrm{CH}_{2}-\mathrm{Th},{ }^{3} J_{\mathrm{H}-\mathrm{H}}=7.9 \mathrm{~Hz}\right), 3.42\left(\mathrm{t}, 2 \mathrm{H}, \mathrm{CH}_{2}-\mathrm{Br}\right.$, $\left.{ }^{3} J_{\mathrm{H}-\mathrm{H}}=6.7 \mathrm{~Hz}\right), 6.98\left(\mathrm{~s}, 2 \mathrm{H}\right.$, Th) ppm. ${ }^{13} \mathrm{C}\left\{{ }^{1} \mathrm{H}\right\} \mathrm{NMR}\left(\mathrm{CDCl}_{3}\right)$ : 14.3, 22.8, 28.1, 28.8, 29.4, 29.6, 30.5, 30.7, 31.8, 32.9, 34.0, 128.8, 130.7, 130.8, 139.8, 140.0. UV-Vis $\left(\mathrm{CHCl}_{3}\right): \lambda_{\max }=450$ $\mathrm{nm}$; SEC (THF, PS standards): $\mathrm{M}_{\mathrm{n}}=11000 \mathrm{~g} \mathrm{~mol}^{-1}, \mathrm{M}_{\mathrm{w}}=$ $12300 \mathrm{~g} \mathrm{~mol}^{-1}$, PDI $=1.12$.

General procedure for the synthesis of P3HT-b-P3HTIm and P3HT-b-P3HTPy block copolyelectrolytes. Block copolymer P3HT-b-P3HTBr $(150 \mathrm{mg})$ was allowed to react with 1-methylimidazole $(10 \mathrm{~mL})$ or pyridine $(10 \mathrm{~mL})$ in refluxing $\mathrm{CHCl}_{3}(10 \mathrm{~mL})$ for $2 \mathrm{~d}$. After cooling to room temperature, the mixture was poured into $\mathrm{Et}_{2} \mathrm{O}$ to precipitate the polyelectrolytes P3HT-b-P3HTIm and P3HT-b-P3HTPy. The crude polymers obtained were repeatedly washed with diethyl ether to remove excess of 1-methylimidazole or pyridine, and then dried under vacuum at $40^{\circ} \mathrm{C}$. The solid polymers P3HT- $b$ P3HTIm and P3HT-b-P3HTPy were further purified in refluxing diethyl ether using a Soxhlet apparatus overnight and finally, dried under vacuum at $40^{\circ} \mathrm{C}$.

P3HT-b-P3HTIm. Yield: $80 \%,{ }^{1} \mathrm{H}$ NMR $\left(\mathrm{CDCl}_{3}\right): \delta=0.89$ (br.), 1.19-1.47 (br.), 1.69 (br.), 1.90 (br.), 2.78 (br.), 3.19 (br.), 4.05 (br.), 4.34 (br.), 6.97 (br.), 7.74 (br.), 9.92 (br.) ppm. ${ }^{13} \mathrm{C}\left\{{ }^{1} \mathrm{H}\right\} \mathrm{NMR}\left(\mathrm{CDCl}_{3}\right): \delta=14.2,22.8,26.3,29.1,29.4,29.6$, $30.5,30.6,31.8,36.9,50.1,122.8,124.1,128.7,130.6,133.8$, 136.7, $140.0 \mathrm{ppm}$. UV-Vis $\left(\mathrm{CHCl}_{3}\right): \lambda_{\max }=452 \mathrm{~nm}$; Anal. calcd.: N/S 0.86 Found 0.86.

P3HT-b-P3HTPy. Yield: $81 \%,{ }^{1} \mathrm{H}$ NMR $\left(\mathrm{CDCl}_{3}\right): \delta=0.91$ (br.), 1.27-1.50 (br.), 1.70 (br.), 2.04 (br.), 2.80 (br.), 4.00 (br.), 6.96 (br.), 8.16 (br.), 8.60 (br.), 9.50 (br.) ppm, ${ }^{13} \mathrm{C}\left\{{ }^{1} \mathrm{H}\right\} \mathrm{NMR}$ $\left(\mathrm{CDCl}_{3}\right): \delta=15.4,23.8,27.1,30.2,30.5,30.7,31.7,32.8,33.2$, 
$62.9,129.8,131.7,134.9,141.1,146.3147 .0$ ppm. UV-Vis $\left(\mathrm{CHCl}_{3}\right): \lambda_{\max }=455 \mathrm{~nm}$; Anal. calcd.: N/S 0.43 Found 0.41.

General procedure for the synthesis of P3HT- $b$-P3HTNMе and P3HT-b-P3HTPMe 3 block copolyelectrolytes. Block copolymer P3HT-b-P3HTBr (150 mg) was introduced into a $100 \mathrm{~mL}$ two-necked flask equipped for stirring and dissolved in $\mathrm{CHCl}_{3}(15 \mathrm{~mL}) .12 \mathrm{~mL}$ of a solution of trimethylamine or trimethylphosphine (1.0 M in THF) were added and the mixture was stirred at $40^{\circ} \mathrm{C}$ for $48 \mathrm{~h}$. After cooling to room temperature, the solvent mixture was evaporated and the residue was dissolved in the minimum amount of $\mathrm{CHCl}_{3}$ and poured into $\mathrm{Et}_{2} \mathrm{O}$ to precipitate the polyelectrolytes P3HT-b-P3HTNMe $\mathbf{H}_{3}$ or P3HT-b-P3HTPMe 3 . The crude products obtained were repeatedly washed with diethyl ether and dried under vacuum at $40^{\circ} \mathrm{C}$. P3HT-b-P3HTNMе ${ }_{3}$ and P3HT-b-P3HTPMe $\mathbf{e}_{3}$ were further purified with refluxing diethyl ether using a Soxhlet apparatus overnight and finally, dried under vacuum at $40^{\circ} \mathrm{C}$.

P3HT-b-P3HTNMe 3 . Yield: 79\%, ${ }^{1} \mathrm{H}$ NMR $\left(\mathrm{CDCl}_{3}\right): \delta=0.90$ (br.), 1,15-1.48 (m, br.), 1.71 (m, br.), 2.80 (br.), 3.35 (br.), 3.64 (br.) 6.95 (br.) ppm, ${ }^{13} \mathrm{C}\left\{{ }^{1} \mathrm{H}\right\} \mathrm{NMR}\left(\mathrm{CDCl}_{3}\right)$ : 14.2, 22.8, 29.4, 29.6, 30.6, 31.8, 66.5 128.7, 130.6, 133.8, 134.2, 140.0 ppm. UV-Vis $\left(\mathrm{CHCl}_{3}\right): \lambda_{\max }=452 \mathrm{~nm}$; Anal. calcd.: N/S 0.43 Found 0.40 .

P3HT- $\boldsymbol{b}$-P3HTPMe 3 . Yield: $80 \%,{ }^{1} \mathrm{H}$ NMR $\left(\mathrm{CDCl}_{3}\right)$ : $\delta=0.90$ (br.), 1.24-1.48 (br.), 1.70 (br.), 1.91 (br.), 2.12 (br.), 2.56 (br.), 2.80 (br.), 6.97 (br.) ppm. ${ }^{13} \mathrm{C}\left\{{ }^{1} \mathrm{H}\right\} \mathrm{NMR}\left(\mathrm{CDCl}_{3}\right): \delta=10.0$, 10.3, 15.2, 22.8, 23.6, 23.8, 30.4, 30.2, 30.7, 31.4, 31.7, 32.7, $32.9,129.7,131.7,134.8,141.0 \mathrm{ppm} .{ }^{31} \mathrm{P}\left\{{ }^{1} \mathrm{H}\right\} \mathrm{NMR}\left(\mathrm{CDCl}_{3}\right): \delta$ $=27.0(\mathrm{~s}, 1 \mathrm{P})$ ppm. UV-Vis $\left(\mathrm{CHCl}_{3}\right): \lambda_{\max }=455 \mathrm{~nm}$.

\section{Results and discussion}

\section{Polymer Synthesis}

The cationic diblock copolythiophenes were synthesised by a two-step procedure, similar to the analogous random copolymers and homopolymers. ${ }^{8,14}$ In the first step, the preparation of the regioregular head-to-tail bromide-bearing copolythiophene (P3HT-b-P3HTBr) in a 50/50 block ratio is achieved by using KTCP polymerisation (Scheme 1). ${ }^{24,34}$

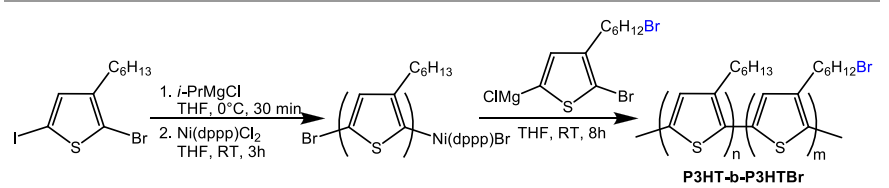

Scheme 1. Synthetic route applied toward the $\mathbf{P} 3 \mathbf{H T}-\boldsymbol{b}-\mathbf{P} \mathbf{3} \mathbf{H T B r}$ precursor block copolymer.

The P3HT block was first synthesised by polymerisation of 2-bromo-3-hexyl-5-iodothiophene with the Ni catalyst to obtain the end-living P3HT polymer. Subsequently, a new portion of activated 2,5-dibromo-3-bromohexylthiophene monomer was added to the reacting solution to obtain the final diblock copolymer P3HT-b-P3HTBr. After the polymerisation, the reaction mixture was quenched with $5 \mathrm{M} \mathrm{HCl}$, following the procedure described by Yokozawa et $a .^{35}$ This quenching procedure prevents the polymer chains from undergoing dimerisation, in order to maintain the narrow polydispersity while efficiently removing inorganic impurities from the product.

Figure 1 shows the SEC profiles of the P3HT block synthesised in the first step and the final diblock copolymer P3HT-b-P3HTBr after the second-stage polymerisation. Compared to the elution profile of the P3HT block obtained after the first step, the peak of the final diblock copolythiophene shifts to shorter elution volume (corresponding to a higher molecular weight), whilst maintaining a single peak, indicating the 'living' character and the formation of the diblock copolymer. A number-averaged molecular weight $\left(\mathrm{M}_{\mathrm{n}}\right)$ of $11000 \mathrm{~g} \mathrm{~mol}^{-1}$ and a polydispersity index $\left(\mathrm{M}_{\mathrm{w}} / \mathrm{M}_{\mathrm{n}}\right)<1.3$ were obtained from the monomodal trace of the P3HT-b-P3HTBr copolymer in the SEC analysis.

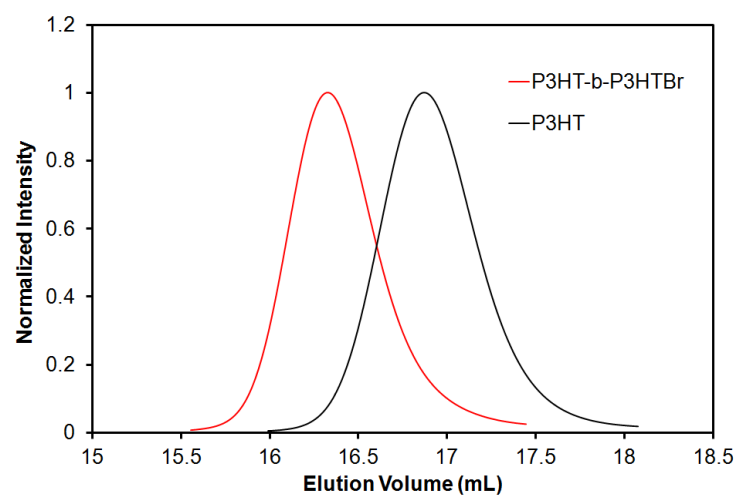

Figure 1. SEC profiles obtained during the synthesis of the P3HT-b-P3HTBr block copolymer.

MALDI-ToF analysis of P3HT- $\boldsymbol{b}$-P3HTBr confirmed the covalent association of 3-bromohexylthiophene and 3hexylthiophene. Indeed, the presence of $\mathrm{m} / \mathrm{z} 166$ and $\mathrm{m} / \mathrm{z} 244$ mass differences confirmed the presence of both monomer units inside the polymer chains (see Figure S1 in Supporting Information). However, the exact nature of the end-groups cannot be determined since, for instance, $\mathrm{H}(3 \mathrm{HT})_{\mathrm{x}}-(3 \mathrm{HTBr})_{\mathrm{y}}-\mathrm{Br}$

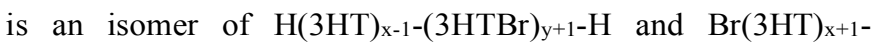

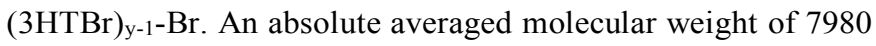
$\mathrm{g} \mathrm{mol}^{-1}$ was determined by MALDI. Such difference between the molecular weight calculated by SEC and MALDI is not unusual since GPC usually overestimates the molecular weight of rigid-rod poly(3-alkylthiophenes) by a factor of 1.2-2.3..$^{20,36}$ The composition of the diblock copolythiophene was determined from the ${ }^{1} \mathrm{H}$ NMR spectra (Figure S2). The peaks observed at $\delta 0.90$ and $3.42 \mathrm{ppm}$ have been assigned to the resonance of the terminal methyl groups in the P3HT block and the methylene groups adjacent to the bromine atoms in the P3HTBr block, respectively. From the integration of these two peaks, the molar ratios of the P3HT and P3HTBr segments was 58:42, which is very close to the feed molar ratio of 55:45. 


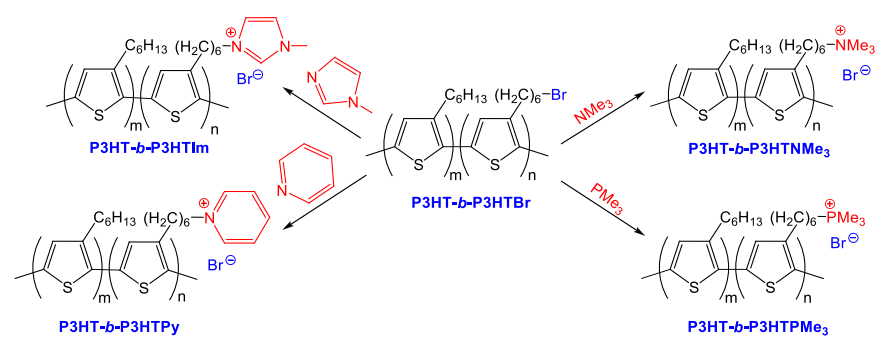

Scheme 2. Synthesis of the polyelectrolyte diblock copolymers P3HT-b-CPE (CPE

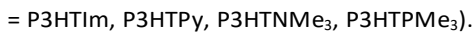

The final step towards the diblock copolyelectrolytes P3HT-b-CPE involved the quaternization of the P3HT-bP3HTBr precursor with 1-methyl-imidazole, pyridine, trimethylamine or trimethylphosphine (Scheme 2). The efficiency of the conversion was monitored by ${ }^{1} \mathrm{H}$ NMR spectroscopy by following the shift of the terminal methylene group $\alpha$ of bromine at $\sim 3.4 \mathrm{ppm}$ (in $\mathrm{CDCl}_{3}$ ) for the precursor copolymer. Based on ${ }^{1} \mathrm{H}$ NMR spectroscopy, the quaternisation appears to be quantitative. It was also confirmed by the N/S ratio values obtained from elemental analysis for the ionic diblock copolymers containing cationic nitrogen side groups which are very close to the expected values calculated on the basis of the composition of the precursor diblock copolymer.

\section{Thermal properties}

In Rapid Heat-Cool (RHC) calorimetry measurements, an important endothermal effect can be observed during the first heating of all four materials, indicating the presence of a significant amount of water or solvent that evaporates upon heating. Such an effect is no longer present during the second heating, where a glass transition temperature $\left(\mathrm{T}_{\mathrm{g}}\right)$ significantly above room temperature is observed for the four materials. The endothermal effect is appearing again when the copolymers are stored in the non-hermetic RHC crucibles at ambient conditions, proving water vapour absorption and indicating the materials' hydrophilicity. Figure 2 shows this behavior for the

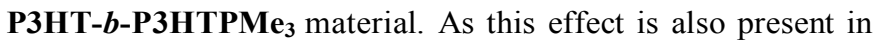
the pure $\mathbf{P 3 H T P M e} 3$ polymer, it can be concluded that it is caused by the CPE block and can be linked to the bromide counterion. ${ }^{14}$ This is an important finding for potential applications, as water vapour absorption will drastically lower the $\mathrm{T}_{\mathrm{g}}$ of this block if the material is not used under dry conditions. ${ }^{37}$

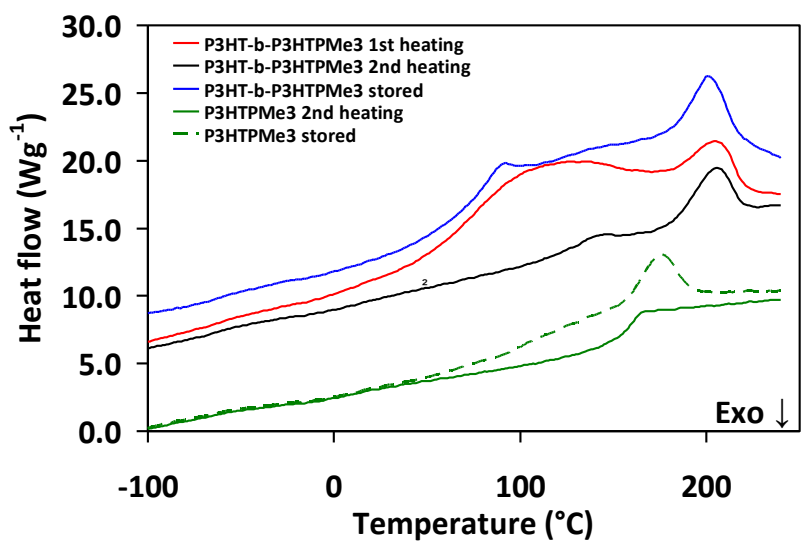

Figure 2. RHC thermogram of P3HT- $b$-P3HTPMe and the second heating (black). After storage at ambient conditions a curve similar to that of the first heating is found (blue). Thermograms of the second heating and after storage at ambient conditions are also included for pure $\mathrm{P3HTPMe}_{3}$ for comparison (dashed and solid green). The curves were shifted vertically for clarity.

When comparing the second heating runs of the different P3HT-b-CPE polymers, a melting peak is observed at a similar temperature (around $205^{\circ} \mathrm{C}$ ) for all materials except P3HT- $b$ P3HTNMe $_{3}$. These melting transitions can be attributed to the P3HT block, which is known to be a fast-crystallising conjugated polymer. A clear $\mathrm{T}_{\mathrm{g}}$ is also always present, caused by the CPE block, as can be seen by comparing with the pure CPE materials. The CPE block $T_{g}$ and the melting point $\left(T_{m}\right)$ and enthalpy $\left(\Delta \mathrm{H}_{\mathrm{m}}\right)$ of the P3HT block are summarised for the four copolymers in Table 1.

Table 1. Glass transition of the CPE block and melting temperature and enthalpy of the P3HT block for all P3HT- $\boldsymbol{b}$-CPE materials measured by RHC during the second heating run.

\begin{tabular}{ccc} 
Polymer & $\mathrm{T}_{\mathrm{g}}\left({ }^{\circ} \mathrm{C}\right)$ & $\mathrm{T}_{\mathrm{m}}\left({ }^{\circ} \mathrm{C}\right) / \Delta \mathrm{H}_{\mathrm{m}}\left(\mathrm{J} . \mathrm{g}^{-1}\right)$ \\
\hline P3HT-b-P3HTIm & 57 & $206 / 13.1$ \\
P3HT-b-P3HTPy & 75 & $204 / 10.2$ \\
P3HT-b-P3HTNMe $_{3}$ & $159^{*}$ & $177 / 7.3$ \\
P3HT-b-P3HTPMe $_{3}$ & 136 & $205 / 9.2$
\end{tabular}

* Due to the overlap with the melting peak of the P3HT block, the Tg of the $\mathrm{P}_{3} \mathrm{HTNMe}_{3}$ block could not be determined in the block copolymer; instead, the value of the pure $\mathrm{P}_{3} \mathrm{HTNMe}_{3}$ polymer is mentioned.

The thermal transitions observed by RHC during the second heating run are a confirmation of the diblock copolymer architecture of the synthesized materials. The nature of the CPE block can modify its $\mathrm{T}_{\mathrm{g}}$ by approximately $100^{\circ} \mathrm{C}$, ranging from $57^{\circ} \mathrm{C}$ up to $159^{\circ} \mathrm{C}$. The thermogram of P3HT-b-P3HTNMe (Figure 3) clearly shows that the P3HT melting transition differs for this material. 


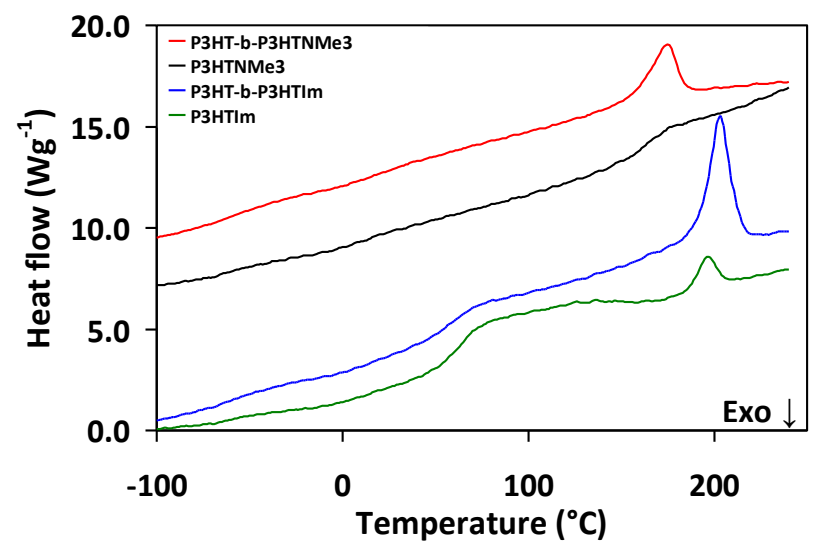

Figure 3. RHC thermogram of $\mathrm{P} 3 \mathrm{HT}-\boldsymbol{b}$ - $\mathrm{P}_{3} \mathrm{HTNM}_{3}$ (red) and $\mathrm{P} 3 \mathrm{HT}-\boldsymbol{b}$ - $\mathrm{P} 3 \mathrm{HTIm}$ (blue), showing the second heating. For comparison the second heating of the $\mathrm{P}_{3} \mathrm{HTNMe}_{3}$ (black) and P3HTIm (green) pure polymers is shown. The curves were shifted vertically for clarity.

In this case, the $\mathrm{T}_{\mathrm{g}}$ of the P3HTNMe $\mathbf{P}_{3}$ block overlaps with the P3HT melting peak, and this high $\mathrm{T}_{\mathrm{g}}$ value around $159^{\circ} \mathrm{C}$ is expected to strongly inhibit the crystallization process of the P3HT block. Indeed, in the temperature region of P3HT crystallization during cooling, the P3HTNMe $_{3}$ block can impose severe mobility restrictions by a concurrent vitrification. This results in a lower melting temperature and enthalpy of the P3HT block. On the contrary, the other P3HTb-CPE copolymers do not show this behaviour, such as e.g. P3HT-b-P3HTIm (see Figure 3). Interestingly, the pure P3HTIm polymer seems to be somewhat crystalline, showing a small melting peak at about $196^{\circ} \mathrm{C}$ (in close vicinity of the P3HT melting). This explains the slightly higher melting enthalpy found for the P3HT-b-P3HTIm copolymer, when compared to the other materials. It should be noted that the thermograms of Figure 3 and the values of Table 1 are indicative and demonstrate the trends of the different P3HT-bCPE polymers during the second heating for the applied RHC conditions. The position of $\mathrm{T}_{\mathrm{g}}$ and $\mathrm{T}_{\mathrm{m}}$ and the melting enthalpy $\Delta \mathrm{H}_{\mathrm{m}}$ strongly depend on all experimental conditions, i.e. the synthesis procedure (sequence of block synthesis, precise block length control and resulting PDI of blocks, regioregularity of P3HT, nature of end-groups), the purifications and the eventual drying (vacuum at $40^{\circ} \mathrm{C}$ ) and storage. Moreover, the solvent dependent assembly of the block copolyelectrolytes and the persistence of the solvent-induced nanomorphology after removal of the solvent are probably important for the thermal properties and their stability as well (see further in 'Aggregation behaviour' as studied by DLS, SANS, AFM). As an example, the thermal properties of P3HT-b-P3HTIm are still evolving in subsequent cool-heat cycles after the second heating; $\mathrm{T}_{\mathrm{g}}$ is raised from $57^{\circ} \mathrm{C}$ to above $70^{\circ} \mathrm{C}$ after 10 cycles, while $\mathrm{T}_{\mathrm{m}}$ is decreasing from $206^{\circ} \mathrm{C}$ towards $180^{\circ} \mathrm{C}$ in combination with a lowered $\Delta \mathrm{H}_{\mathrm{m}}$ from $13.1 \mathrm{~J} \mathrm{~g}^{-1}$ to $8.5 \mathrm{~J} \mathrm{~g}^{-1}$. These findings suggest further changes in the nanomorphology as a result of thermal cycling, leading to a loss of perfection of the P3HT crystals, possibly with a concomitant loss of a minor amount of residual solvent from the ionic block. Similar effects are noticed for the other P3HT-b-CPE polymers.

\section{Solution behaviour}

The optical properties of the cationic P3HT-b-CPE copolymers in solution have been studied by UV/Vis absorption and photoluminescence (PL) spectroscopy. The neutral diblock copolymer P3HT-b-P3HTBr exhibits a broad absorption band with a maximum $\left(\lambda_{\max }\right)$ centred at $450 \mathrm{~nm}$ in chloroform $\left(\mathrm{CHCl}_{3}\right)$, which, as expected, is similar to the parent P3HT. After functionalization with the imidazolium, pyridinium, trimethylammonium or trimethylphosphonium moieties, the P3HT-b-CPE polymers become also soluble in more polar solvents such as water and $\mathrm{MeOH}$ due to the presence of the hydrophilic pendant ionic side groups which overcomes the hydrophobic $\pi-\pi$ stacking interactions between the polythiophene backbones. We note that P3HT-b-P3HTPy is somewhat less soluble in these solvents than its P3HT-b-CPE counterparts, indicating the somewhat reduced hydrophilic (or increased hydrophobic) character of the P3HTPy block, as previously reported by Scherf et al. for the polyfluorene (PF2/6)-polythiophene diblock copolyelectrolyte analogue PF2/6-P3HTPy. ${ }^{12 a}$ P3HT-b-CPE copolymers exhibit solvatochromatic behaviour, appearing dark red/brown in $\mathrm{CHCl}_{3}$ and dark violet in methanolic and aqueous solution (Fig. 4a and Fig. S13-S15). This solvatochromatism is reflected in the corresponding UV/Vis absorption spectra, as shown in Fig. $4 \mathrm{~b}$ for P3HT- $\boldsymbol{b}$-P3HTPMe 3 where $\lambda_{\max }$ red-shifts from $\sim 450$ $\mathrm{nm}$ in $\mathrm{CHCl}_{3}$, to $\sim 520 \mathrm{~nm}$ in $\mathrm{H}_{2} \mathrm{O}$ and $\mathrm{MeOH}$, with the additional emergence of a vibronic structure in the latter solvents.
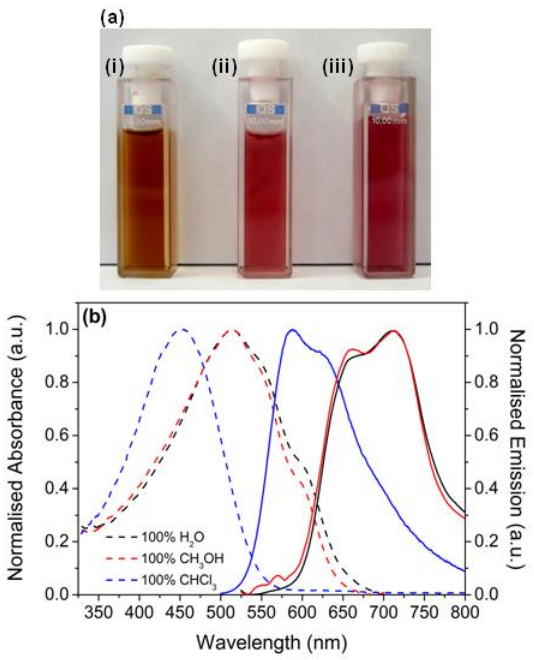

Figure 4. (a) $\mathrm{P3HT}-b$ - $\mathrm{P}_{3} \mathrm{HTPMe}_{3}$ in (i) $\mathrm{CHCl}_{3}$, (ii) $\mathrm{MeOH}$ and (iii) $\mathrm{H}_{2} \mathrm{O}$. (b) Corresponding normalised UV/Vis absorption and emission spectra for P3HT- $\boldsymbol{b}$ $\mathrm{P}_{3} \mathrm{HTPMe}_{3}$ in $100 \% \mathrm{CHCl}_{3}$ (blue), $100 \% \mathrm{H}_{2} \mathrm{O}$ (black) and $100 \% \mathrm{MeOH}$ (red).

P3HT has previously been reported to self-assemble into a lamellar-like structure built from interchain $\pi$ - $\pi$ stacking interactions between the planarised aromatic backbones of the polymer chains. ${ }^{38}$ This supramolecular organisation is 
accompanied by a bathochromic shift in the absorption spectrum and vibronic structure at longer wavelengths, very similar to the behaviour observed here for P3HT-b-CPEs in $\mathrm{MeOH}$ and water. Water is expected to be a non-solvent for the hydrophobic, neutral P3HT block, with only low to moderate solubility anticipated in $\mathrm{MeOH}$. We propose that the significant bathochromic shift is indicative of increased aggregation of the hydrophobic P3HT block in these poor solvents. The concomitant appearance of vibronic structure further suggests increased confinement and/or planarisation of one or both of the blocks, thereby hindering free rotation of thiophene units along the polymer backbone. The P3HT-b-CPE copolymers are weakly fluorescent in solution and the emission spectra exhibit similar solvatochromatic trends (Fig. 4b). A significant red-shift in the emission maximum $\left(\lambda_{\mathrm{em}}\right)$ from $588 \mathrm{~nm}$ to 711 $\mathrm{nm}$ is observed for P3HT-b-P3HTPMe 3 upon changing the solvent from $\mathrm{CHCl}_{3}$ to $\mathrm{MeOH}$ or $\mathrm{H}_{2} \mathrm{O}$, which is again accompanied by increased vibronic structure assigned to the vibronic progression of the $\mathrm{C}=\mathrm{C}$ stretching mode $(\Delta E \approx 0.15$ $\mathrm{eV}) .{ }^{39}$ The optical properties are similar for all copolymers in the P3HT-b-CPE series and absorption and photoluminescence maxima in each solvent are collected in Table 2. The use of solvent mixtures has been shown to be very effective for both breaking up the aggregates formed by homopolymer CPEs in aqueous solution ${ }^{40}$ and controlling segregation and selforganisation of diblock copolymers. ${ }^{12,13}$

\begin{tabular}{lcccccc}
\hline \multirow{2}{*}{ Polymer } & \multicolumn{3}{c}{ Absorption $\left(\lambda_{\max } / \mathrm{nm}\right)$} & \multicolumn{3}{c}{ Emission $\left(\lambda_{\max } / \mathrm{nm}\right)$} \\
\cline { 2 - 7 } & $\mathrm{CHCl}_{3}$ & $\mathrm{MeOH}$ & $\mathrm{H}_{2} \mathrm{O}$ & $\mathrm{CHCl}_{3}$ & $\mathrm{MeOH}$ & $\mathrm{H}_{2} \mathrm{O}$ \\
\hline P3HT-b-P3HTIm & 452 & 519 & 522 & 586 & 729 & 729 \\
& & 556 & & 625 & & \\
P3HT-b-P3HTPy $^{4}$ & 455 & 517 & 520 & 580 & 721 & 723 \\
& & 556 & & 619 & & \\
P3HT-b-P3HTNMe $_{3}$ & 452 & 518 & 518 & 592 & 721 & 722 \\
& & 556 & & 631 & & \\
P3HT-b-P3HTPMe $_{3}$ & 455 & 607 & & & & \\
& & 516 & 516 & 588 & 707 & 710 \\
& & 607 & & 627 & & \\
\end{tabular}

Table 2. Absorption and photoluminescence maxima of the P3HT- $\boldsymbol{b}$-CPE diblock copolymers in various solvents. (PL excitation wavelength: $523 \mathrm{~nm}$ )

The UV/Vis absorption and PL properties of P3HT- $b$-CPE copolymers were thus further examined in $\mathrm{MeOH} / \mathrm{H}_{2} \mathrm{O}$ solvent mixtures from $0-100 \%(\mathrm{v} / \mathrm{v})$, as illustrated in Fig. 5 for P3HTb-P3HTPy and in Fig. S16-S18 for other P3HT-b-CPEs. The gradual increase in the volume of $\mathrm{MeOH}$ added to an aqueous solution of P3HT-b-P3HTPy is accompanied by a concomitant increase in the absorbance, which reaches its maximum value at $50 \%(\mathrm{v} / \mathrm{v}) \mathrm{MeOH} / \mathrm{H}_{2} \mathrm{O}$; above this, the absorbance drops off once again (Fig. 5a, inset). A similar trend is observed in the corresponding PL spectrum, with the emission intensity again reaching its upper limit at 50\% (v/v) $\mathrm{MeOH} / \mathrm{H}_{2} \mathrm{O}$ (Fig. 5b). Notably, no shift in the absorption or emission maximum wavelength was observed for any of the solvent mixtures studied.

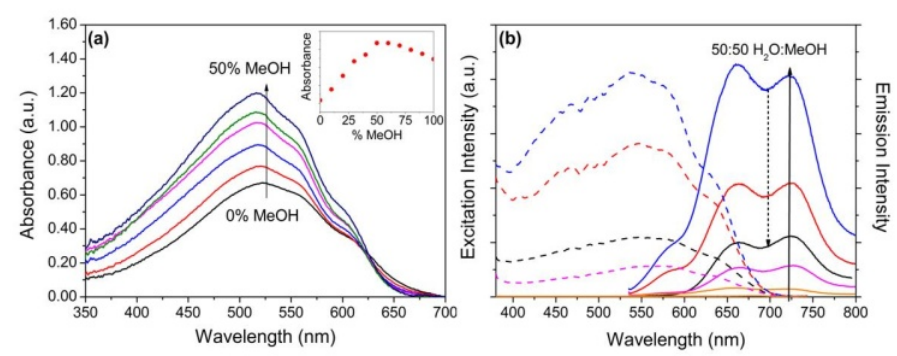

Figure 5. (a) UV/Vis absorption spectra of P3HT-b-P3HTPy in $\mathrm{H}_{2} \mathrm{O} / \mathrm{MeOH}$ solvent mixtures. Inset: Absorbance at $522 \mathrm{~nm}\left(\lambda_{\max }\right)$ as a function of $\mathrm{Vol} \% \mathrm{MeOH}$ in $\mathrm{H}_{2} \mathrm{O} / \mathrm{MeOH}$ mixtures. (b) Selected excitation $\left(\lambda_{\mathrm{em}}=730 \mathrm{~nm}\right)$ and $\mathrm{PL}\left(\lambda_{\mathrm{ex}}=515 \mathrm{~nm}\right)$ spectra of P3HT-b-P3HTPy in $100 \% \mathrm{MeOH}$ (orange line), $70 \% \mathrm{MeOH}$ (violet line), $50 \% \mathrm{MeOH}$ (blue line), $30 \% \mathrm{MeOH}$ (red line) and $0 \% \mathrm{MeOH}$ (black line).

Fluorescence quantum yields $\left(\Phi_{\mathrm{F}}\right)$ were determined to quantify the extent of the fluorescence enhancement in each solvent mixture (Fig. 6a). For P3HT-b-P3HTPy, P3HT-bP3HTNMe $_{3}$ and P3HT-b-P3HTPMe $3, \quad \Phi_{\mathrm{F}} \sim 0.5 \%$ in $100 \%$ $\mathrm{MeOH}$ and $\Phi_{\mathrm{F}} \sim 1.0-1.5 \%$ in $100 \% \mathrm{H}_{2} \mathrm{O}$ were obtained. As the ratio of solvents in the mixture is varied, $\Phi_{\mathrm{F}}$ gradually increases, reaching a maximum of $\Phi_{\mathrm{F}} \sim 5.5 \%$ in $50 \%(\mathrm{v} / \mathrm{v})$ $\mathrm{MeOH} / \mathrm{H}_{2} \mathrm{O}$. P3HT-b-P3HTIm exhibits a slightly different trend; $\Phi_{\mathrm{F}}$ remains constant at $\sim 1.0-1.5 \%$ across the range $100 \%$ $\mathrm{MeOH}$ to $20: 80 \%(\mathrm{v} / \mathrm{v}) \mathrm{MeOH} / \mathrm{H}_{2} \mathrm{O}$, before doubling to $\Phi_{\mathrm{F}} \sim 3 \%$ in $100 \% \mathrm{H}_{2} \mathrm{O}$. The increase in the fluorescence quantum yield in the mixed solvent system suggests improved solubilisation and at least, partial isolation of polymer chains in one or both the blocks.
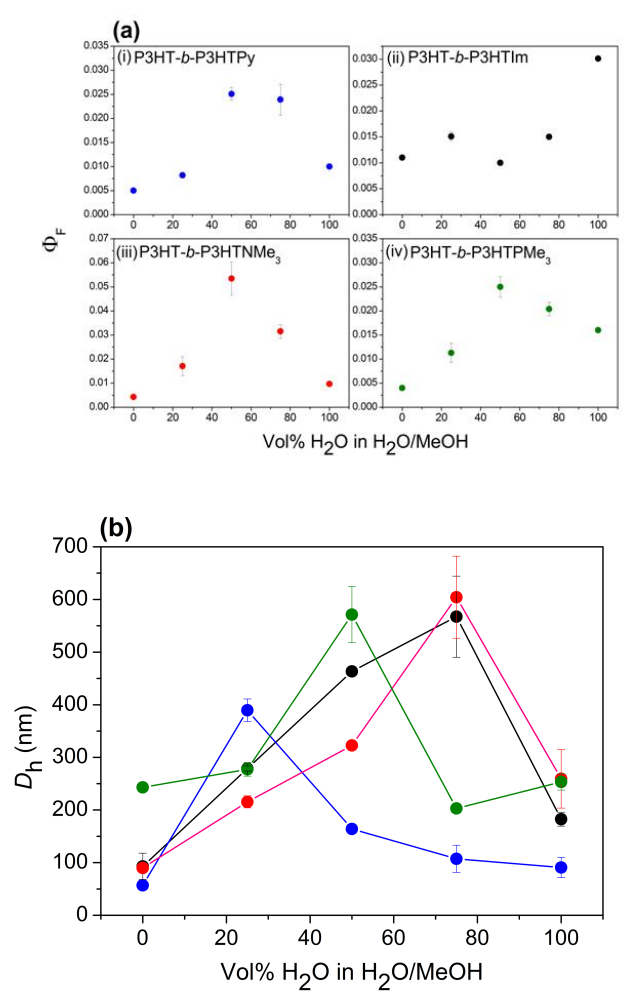
Figure 6. (a) Fluorescence quantum yields $\left(\Phi_{\mathrm{F}}\right)$ and (b) hydrodynamic diameter ( $D_{\mathrm{h}}, \mathrm{Z}$-average) of P3HT-b-CPEs in $\mathrm{H}_{2} \mathrm{O} / \mathrm{MeOH}$ solvent mixtures $(0.35 \mu \mathrm{g} \mathrm{mL}-1)$. Legend: P3HT- $\boldsymbol{b}$-P3HTPy (blue circles), P3HT- $\boldsymbol{b}$-P3HTIm (black circles), P3HT- $\boldsymbol{b}$ $\mathbf{P 3 H T N M e}_{3}$ (red circles) and P3HT-b-P3HTPMe ${ }_{3}$ (green circles).

\section{Aggregation Behaviour}

The aggregation behaviour of P3HT-b-CPEs in solution and thin films has been further investigated by dynamic light scattering (DLS), small-angle neutron scattering (SANS) and atomic force microscopy (AFM). DLS analysis was initially performed to determine the average hydrodynamic diameter $\left(D_{\mathrm{h}}\right)$ of P3HT-b-CPE aggregates in $\mathrm{MeOH} / \mathrm{H}_{2} \mathrm{O}$ mixtures (Fig. $6 b)$. Samples typically exhibited a monomodal distribution of low polydispersity in all solvent mixtures investigated. The smallest aggregates were obtained in $100 \% \mathrm{MeOH}(\sim 100 \mathrm{~nm}$ for P3HT- $b$-P3HTNMе 3 , P3HT- $b$-P3HTIm, P3HT- $b$ P3HTPy and $\sim 250 \mathrm{~nm}$ for P3HT-b-P3HTPMe 3 ), with slightly larger aggregates found in $100 \% \mathrm{H}_{2} \mathrm{O}(\sim 100 \mathrm{~nm}$ for P3HT- $\boldsymbol{b}$ P3HTPy, 200-260 nm for P3HT-b-P3HTIm and P3HT-bP3HTPMe $_{3}$, and $\sim 300 \mathrm{~nm}$ P3HT-b-P3HTNMe $)$. The largest $D_{\mathrm{h}}$ values $(\sim 400-600 \mathrm{~nm})$ were consistently obtained in $\sim 40$ $60 \%(\mathrm{v} / \mathrm{v}) \mathrm{MeOH} / \mathrm{H}_{2} \mathrm{O}$, which is consistent with increased solubility of both the neutral P3HT block and the charged CPE block in the mixed solvent systems, resulting in swelling of the aggregate structure due to associated solvent molecules.

To obtain deeper insight into the particle shape and nanoscale organisation in solution, small-angle neutron scattering (SANS) studies were performed on P3HT-bP3HTIm and P3HT-b-P3HTPy in deuterated solution. Figure 7 plots the SANS data for P3HT-b-P3HTPy in $\mathrm{D}_{2} \mathrm{O}, d_{4}-\mathrm{MeOD}$ and their 50:50 (v/v) mixture (corresponding plots for P3HT-bP3HTIm are shown in Figure S22 in Supporting Information). All three solvents yield similar scattering profiles with a shoulder at $q=0.02 \AA^{-1}$ and an upturn at $q=0.008 \AA^{-1}$; however they do differ in the gradient of their slopes. At high $q$ $\left(q>0.07 \AA^{-1}\right)$, the SANS response stems from the internal structure of the aggregate. The excess scatter for some samples at $q \sim 0.1-0.2 \AA^{-1}$ is likely due to internal structure within the aggregates, perhaps due to repulsive interactions between similarly charged polymer chains. ${ }^{5 \mathrm{a}-\mathrm{c}}$ The upturn in $I(q)$ at $q<0.008 \AA^{-1}$ is indicative of particle aggregation. The power law scaling for the intermediate $q$ region $(0.02<q<0.07)$ varies from $q^{-4.2}$ in $d_{4}-\mathrm{MeOD}$ to $q^{-5.21}$ in $\mathrm{D}_{2} \mathrm{O}$. The low $q$ region $(q<0.02)$ decays as $\sim q^{-5 / 3}$, which is typical of scattering from either cylindrical aggregates or from individual chains. ${ }^{41}$ The observation window of these SANS experiments ranged from 2-160 nm, which is well beyond the isolated chain length (22.7$25.4 \mathrm{~nm}$ ). Unique fits to the SANS data were not possible; however all those chosen (Table S1 in Supplementary Information) have absolute SANS intensities consistent with the known sample concentrations ( $\sim 1 \%$ vol. dry material). All the fits show that the aggregates must contain upwards of $30 \%$ solvent, without any significant "dry core". Better fits were obtained with a core plus shell model with a diffuse outer shell containing $85-95 \%$ solvent. The exception to the latter were the samples in pure MeOD, where a thin shell (of $\sim 2 \mathrm{~nm}$ ) seems to have slightly less solvent ( $65 \%$ vol.) than the core of the aggregate ( $\sim 80 \%$ vol. solvent). The SANS from the aggregates in $\mathrm{MeOD}$ are also more consistent with an elongated, rod-like structure, whilst the others are better fit as discs. The disc cases would also be equally well fitted as core/shell ellipsoids or as rather polydisperse core/shell spheres. There is no clear evidence in the SANS patterns of a sharp core/shell boundary, so a continuous radial variation in solvent composition seems likely, though this would be more difficult to model. Aggregation numbers are likely in the range $\sim 100$ to $\sim 300$ copolymer molecules.

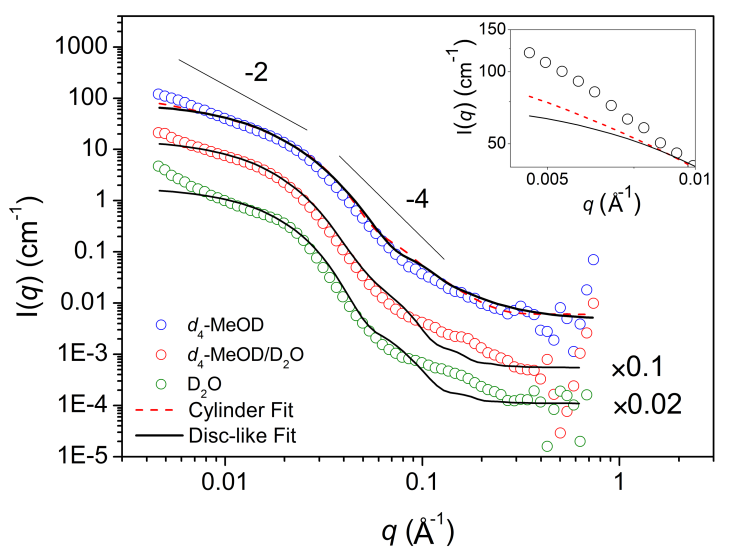

Figure 7. SANS data for P3HT-b-P3HTPy in $d_{4}-\mathrm{MeOD}, 50: 50(\mathrm{v} / \mathrm{v}) d_{4}-\mathrm{MeOD} / \mathrm{D}_{2} \mathrm{O}$ and $\mathrm{D}_{2} \mathrm{O}\left(10 \mathrm{mg} \mathrm{mL}^{-1}\right)$. For clarity, the $d_{4}-\mathrm{MeOD} / \mathrm{D}_{2} \mathrm{O}$ and $\mathrm{D}_{2} \mathrm{O}$ data have been offset by 0.1 and 0.02 , respectively. Straight lines show -2 and -4 decays for comparison. The solid black lines show the disc-like fits obtained using the CoreShell-Cylinder model for each solvent mixture. Only P3HT-b-P3HTPy in $d_{4}$-MeOD was found to be better fit by rods at low $q$, as shown by the red dashed line in the inset.

The SANS data $(0.0046<q<0.6)$ in each solvent were fitted to a Core-Shell-Cylinder model using a non-linear least-squares method, including polydispersity and $q$-resolution smearing (fits shown as solid lines in Figure 7). ${ }^{33}$ For P3HT-b-P3HTPy in $d_{4}$-MeOD, depending on the starting imputed values of length and radius, the fits were found to converge to two slightly differing shapes: (1) a rod with core length, $L_{\text {core, }}$ of $\sim 80 \mathrm{~nm}$, core radius, $r_{\text {core, }}$ of $\sim 6 \mathrm{~nm}$ and shell thickness, $T_{\text {shell, }}$ of $\sim 2 \mathrm{~nm}$; (2) a disc with $L_{\text {core }} \sim 75 \mathrm{~nm}, r_{\text {core }} \sim 30 \mathrm{~nm}$ and $T_{\text {shell }} \sim 1$ $\mathrm{nm}$. The rod-like core-shell aggregate (1) resulted in an improved fit at low $q$ (Figure 7 , inset). The SANS data for P3HT-b-P3HTPy in 50:50 (v/v) $d_{4}-\mathrm{MeOD} / \mathrm{D}_{2} \mathrm{O}$ were also best modelled by a disc-like core-shell aggregate, revealing a wet core with $L_{\text {core }} \sim 11 \mathrm{~nm}$ and $r_{\text {core }} \sim 7.5 \mathrm{~nm}$, and a thick solvated shell $\left(T_{\text {shell }} \sim 9.5 \mathrm{~nm}\right)$. Fitting the SANS data of P3HT- $\boldsymbol{b}$ P3HTPy in $\mathrm{D}_{2} \mathrm{O}$ similarly yielded a disc with a solvated core ( $L_{\text {core }} \sim 9 \mathrm{~nm}, r_{\text {core }} \sim 6 \mathrm{~nm}$ ) and a very wet shell of $T_{\text {shell }} \sim 8 \mathrm{~nm}$. The shell thickness corresponds to the fully extended length of the charged P3HTPy block. Therefore it is likely that the drier core corresponds to the aggregated neutral P3HT blocks. We note that reasonable fits to the SANS data in $\mathrm{D}_{2} \mathrm{O}$ and 50:50 $(\mathrm{v} / \mathrm{v}) d_{4}-\mathrm{MeOD} / \mathrm{D}_{2} \mathrm{O}$ could also be obtained with a Core-Shell 
sphere model, which yielded aggregates of similar dimensions to those described above (Table S2 in Supplementary Information). Similar fits were obtained for P3HT-b-P3HTIm and can be found with the tabulated summaries of the fitting data for both CPEs in the Supporting Information.

The scattering length densities (SLDs) of the neutral and charged CPE blocks are both $\sim 1.1 \times 10^{-6} \AA^{-2}$. Therefore, they can only be distinguished by neutron scattering when one block is substantially more solvated than the other. The SANS data fits for P3HT-b-P3HTPy in $\mathrm{D}_{2} \mathrm{O}$ suggest the presence of aggregates with a highly swollen shell. Since $\mathrm{D}_{2} \mathrm{O}$ is expected to be a selective solvent for the charged CPE block, we propose that the aggregate core consists predominantly of the neutral P3HT blocks, surrounded by a highly solvated CPE shell. Since $\mathrm{D}_{2} \mathrm{O}$ is a non-solvent for P3HT, the hydrophobic effect results in tight packing of the P3HT chains within the aggregate core to minimise interaction with the solvent. In $50: 50(\mathrm{v} / \mathrm{v}) d_{4}-\mathrm{MeOD} / \mathrm{D}_{2} \mathrm{O}$, the core-shell aggregate structure is retained, but the solvent mixture results in improved solvation of both blocks, leading to an increase in both the core radius and shell thickness. In MeOD, P3HT-b-P3HTPy forms elongated core-shell cylindrical structures, with a solvated core and a drier shell. On the basis of the Hildebrand solubility parameters, $\mathrm{MeOH}$ is a relatively poor solvent for P3HT $\left(\delta \sim 19.1 \mathrm{MPa}^{1 / 2}\right.$ for P3HT and $\delta \sim 29.7 \mathrm{MPa}^{1 / 2}$ for $\mathrm{MeOH}$, respectively $\mathrm{y}^{42}$ ) and should be a better solvent for the charged CPE block. We propose that in MeOD, P3HT-b-P3HTPy forms core-shell aggregates in which the P3HT blocks predominantly occupy the core, but cannot exclude partial mixing of the two blocks across both domains. In the future we would like to deuterate the side chains of the P3HT blocks in order to obtain unequivocal evidence for the location of the blocks from SANS.
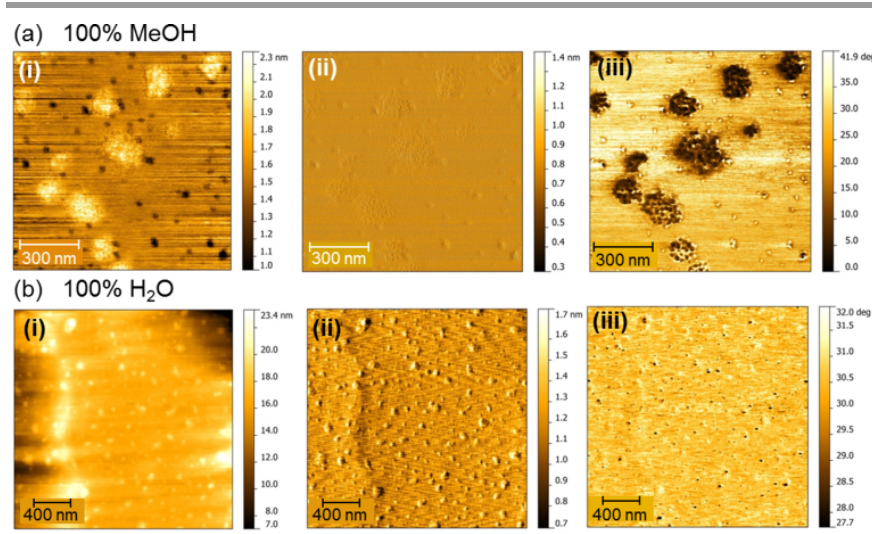

(c) $50 \%(v / v) \mathrm{H}_{2} \mathrm{O} / \mathrm{MeOH}$

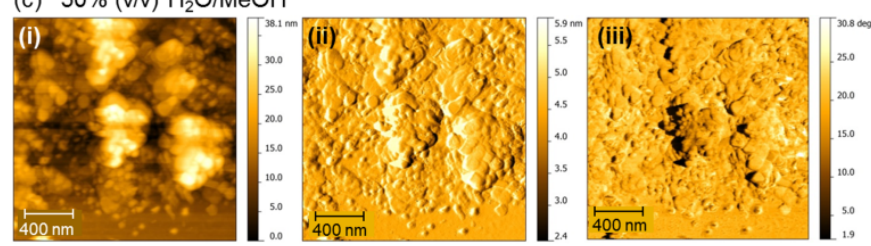

Figure 8 AFM tapping mode images of P3HT-b-P3HTIm films drop-cast from (a) $100 \% \mathrm{MeOH}\left(7 \mu \mathrm{g} \mathrm{mL}^{-1}\right)$, (b) $100 \% \mathrm{H}_{2} \mathrm{O}\left(3.5 \mu \mathrm{g} \mathrm{mL}^{-1}\right)$ and (c) $50 \%(\mathrm{v} / \mathrm{v}) \mathrm{H}_{2} \mathrm{O} / \mathrm{MeOH}$
(3.5 $\mathrm{gg} \mathrm{mL}^{-1}$ ) onto freshly cleaved mica: (i) Height, (ii) amplitude and (iii) phase images, respectively.

Atomic force microscopy was used to determine if the structured aggregates formed in solution could be transferred as thin films with the retention of their nanoscale organisation, which is a key requirement for potential device applications. Solutions of P3HT-b-P3HTIm at low concentrations (3.5-7.0 $\left.\mu \mathrm{g} \mathrm{mL}^{-1}\right)$ drop cast onto mica substrates reveal the occurrence of spheroids whose surface morphology and size critically depend on the deposition solvent. In $\mathrm{MeOH}$, a honeycomb-like texture is observed on the aggregate surface (Figure 8a), with an average diameter of $128 \pm 28 \mathrm{~nm}$. The surface texture may be indicative of the fusion of several smaller structures into larger aggregates. In water, slightly larger, isolated aggregates are observed (average diameter $\sim 147 \pm 28 \mathrm{~nm}$ ), whose surface morphology is much smoother (Figure 8b). In $50 \%(\mathrm{v} / \mathrm{v})$ $\mathrm{MeOH} / \mathrm{H}_{2} \mathrm{O}$, amorphous films formed from extended networks of much smaller, disc-like clusters, $50-80 \mathrm{~nm}$ in diameter, are observed (Figure 8c). Similar trends are observed for all P3HTb-CPEs (Figure S20-21 in Supporting Information). It should be noted that the SANS data indicate that the core-shell aggregates formed in solution are highly solvated; in contrast the corresponding thin film samples were dried prior to AFM imaging. It is conceivable that removal of the solvent during drying may lead to partial collapse or reorganisation of the aggregate structure due to fluctuations in the solvent gradient.

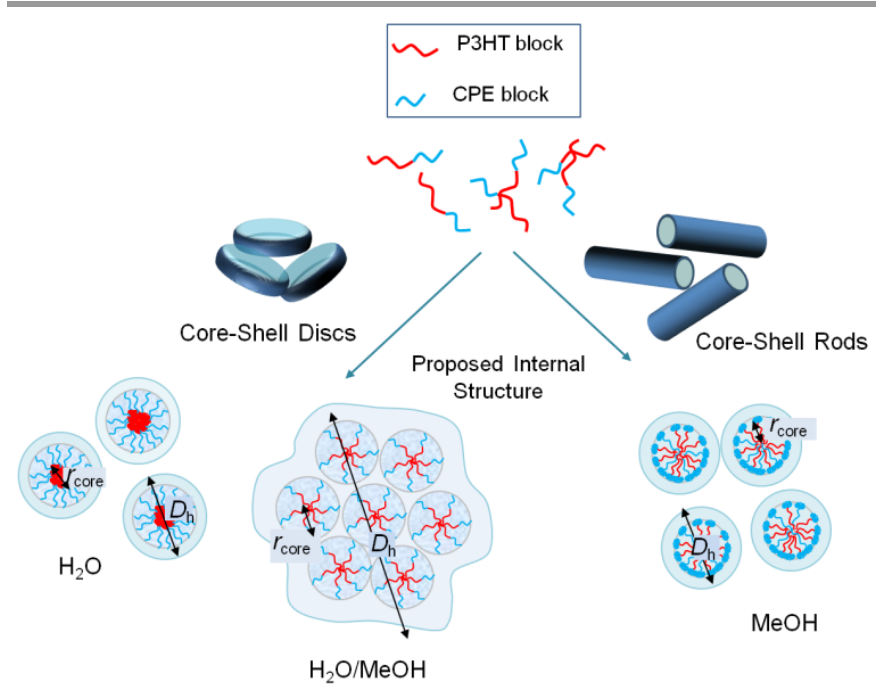

Figure 9. Schematic representation of the proposed internal aggregate structures for P3HT- $b$-CPE in $\mathrm{H}_{2} \mathrm{O}, \mathrm{MeOH}$ and $\mathrm{H}_{2} \mathrm{O} / \mathrm{MeOH}$ mixtures. $L_{\text {core, }} r_{\text {core }}$ and $T_{\text {shell }}$ are the core length, core radius and shell thickness obtained from SANS data fitting, respectively. $D_{\mathrm{h}}$ is the hydrodynamic diameter measured by DLS. We note that the precise arrangement of the $\mathrm{P} 3 \mathrm{HT}$ and CPE block domains in the aggregates formed in $\mathrm{MeOH}$ is still undetermined.

Figure 9 shows a schematic representation of the proposed aggregate structures adopted by P3HT- $\boldsymbol{b}$-CPEs in $\mathrm{H}_{2} \mathrm{O}$, MeOH and $\mathrm{H}_{2} \mathrm{O} / \mathrm{MeOH}$ mixtures. The smallest aggregates are formed in pure methanol (DLS, SANS, AFM) and exhibit a core-shell cylindrical structure comprised of a long, "wet" core, protected by a "dry" thin shell. MeOH is a poor solvent for P3HT, which 
is anticipated to predominantly occupy the aggregate core; however we cannot exclude some mixing of the P3HT and CPE blocks across both the core and shell domains. The small aggregate size results in increased chain proximity and interchain exciton migration, resulting in the lowest $\Phi_{\mathrm{F}}$ values being obtained in this solvent. Slightly larger aggregates are obtained in pure $\mathrm{H}_{2} \mathrm{O}$ (DLS, AFM, SANS) and consist of a drier core of aggregated, neutral P3HT blocks and a wet shell of well-dissolved CPE chains. The largest aggregates are formed in $\mathrm{MeOH} / \mathrm{H}_{2} \mathrm{O}$ mixtures (DLS, SANS), in which the core and shell are both well-solvated. This is in excellent agreement with the corresponding increase in the $\Phi_{\mathrm{F}}$ values observed in $\mathrm{MeOH} / \mathrm{H}_{2} \mathrm{O}$ mixtures. Moreover, due to the larger solubility of the entire CPE chain, we expect lower aggregation numbers for each structure, thereby reducing electrostatic repulsion between the highly-solvated, individual chains from which each aggregate is comprised. Reduced electrostatic repulsion may result in long-range assembly of multiple aggregates, which may explain the large $D_{\mathrm{h}}$ obtained by DLS and the textured extended sheets (rather than individual clusters) observed by AFM.

\section{Conclusions}

Amphiphilic rod-rod diblock copolymers containing a hydrophobic P3HT block and a hydrophilic cationic P3HT block bearing different side groups were synthesized in an efficient way by using a Kumada catalyst-transfer polycondensation. In contrast to the neutral parent polymer P3HT-b-P3HTBr, the incorporation of cationic terminal groups renders P3HT-b-CPEs soluble in highly polar solvents. The thermal properties of these copolymers indicate a clear signature of the diblock architecture by the presence of a $T_{g}$ caused by the CPE block and a $\mathrm{T}_{\mathrm{m}}$ corresponding to the P3HT block. The nature of the CPE block drastically affects the $T_{g}$, and to a lesser extent the melting transition of the P3HT block, except in the case of P3HT-b-P3HTNMe 3 . The UV/Vis absorption and photoluminescence properties are solventdependent, resulting in a significant bathochromic shift in $\lambda_{\max }$, increased vibronic structure and a decrease in the fluorescence quantum yield in pure $\mathrm{MeOH}$ and $\mathrm{H}_{2} \mathrm{O}$, which is attributed to aggregate formation. Fluorescence quantum yields are enhanced in $\mathrm{H}_{2} \mathrm{O} / \mathrm{MeOH}$ mixtures, which are accompanied by an analogous trend in the Z-average particle diameter determined by DLS. Small-angle neutron scattering studies have revealed that P3HT-b-CPEs form core-shell cylindrical aggregates in solution, with the relative thickness and "wetness" of the core and the shell determined by the solvent system. In $\mathrm{H}_{2} \mathrm{O} / \mathrm{MeOH}$ mixtures, we propose that enhanced solvation of both the core and shell results in aggregate swelling, which promotes disaggregation of localised polymer chains, thereby increasing $\Phi_{\mathrm{F}}$. AFM studies reveal that the aggregates formed in solution can be transferred as thin films. This has important implications for the application of these CPEs in organic photovoltaic devices, where efficient charge injection and transport is facilitated by a nanostructured, interpenetrating network at the active interface.

\section{Acknowledgements}

The authors thank the CNRS and the Universite Montpellier II for financial support. This research has been supported by the European Commission under the 7th Framework Programme through the 'Research Infrastructures' action of the 'Capacities' programme. Contract no: CP-CSA_INFRA-2008-1.1.1 Number 226507-NM13. We thank ISIS and STFC for the allocation of SANS Xpress beamtime. Research in Mons is supported by the Science Policy Office of the Belgian Federal Government (BELSPO; PAI 7/05), FNRS-FRFC and Région Wallonne (OPTI2MAT excellence programme). The authors are also grateful to National Fund for Scientific Research (F.R.S.FNRS) in the frame of the FRFC research program (convention No. 2.4508.12). The MS laboratory acknowledges the "Fonds de la Recherche Scientifique (FRS-FNRS)" for its contribution to the acquisition of the Waters QToF Premier Mass Spectrometer. J.D.W. thanks FRS-FNRS (convention No. 2.4508.12) for financial support.

\section{Notes and references}

${ }^{a}$ Institut Charles Gerhardt - UMR 5253, Equipe Chimie Moléculaire et Organisation du Solide, Université Montpellier 2 - CC1701, Place Eugène Bataillon, F-34095 Montpellier Cedex 05, France. E-mail: sebastien.clement02@univ-montp2.fr; Tel: +33467143971.

${ }^{b}$ Laboratory for Chemistry of Novel Materials, Center for Innovation in Materials and Polymers, Research Institute for Science and Engineering of Materials, University of Mons - UMONS, 23 Place du Parc, B-7000 Mons, Belgium.

${ }^{c}$ School of Chemistry, The University of Dublin, Trinity College, Dublin 2, Ireland. E-mail: raevans@tcd.ie

${ }^{d}$ Physical Chemistry and Polymer Science (FYSC), Vrije Universiteit Brussel (VUB), Pleinlaan 2, B-1050 Brussels, Belgium.

${ }^{e}$ Laboratory of organic synthesis and mass spectrometry, Interdisciplinary Centre for Mass Spectrometry, University of Mons-UMONS, 23 Place du Parc, B-7000 Mons, Belgium

$f$ Laboratory for Polymeric and Composites Materials, Center for Innovation in Materials and Polymers, Research Institute for Science and Engineering of Materials, University of Mons - UMONS, 23 Place du Parc, B-7000 Mons, Belgium.

g ISIS-CCLRC, Rutherford Appleton Laboratory, Chilton, Oxon OX11 0QX, United Kingdom.

${ }^{h}$ Centre for Research on Adaptive Nanostructures and Nanodevices (CRANN), Trinity College Dublin, Dublin 2, Ireland.

$\dagger$ Electronic Supplementary Information (ESI) available: ${ }^{1} \mathrm{H},{ }^{13} \mathrm{C}\left\{{ }^{1} \mathrm{H}\right\}$ NMR spectra of the precursor block copolymers and the ionic block copolythiophenes, ${ }^{31} \mathrm{P}\left\{{ }^{1} \mathrm{H}\right\} \quad$ NMR spectra of P3HT-b-P3HTPMe . $_{3}$ MALDI-TOF spectra for P3HT- $\boldsymbol{b}$-P3HTBr. UV-Vis. spectra of P3HT- $\boldsymbol{b}$ CPE. Absorption, excitation and PL spectra of P3HT-b-CPE in $\mathrm{H}_{2} \mathrm{O} / \mathrm{MeOH}$ mixtures. Correlogram and size distribution obtained by DLS of P3HT- $\boldsymbol{b}$-P3HTPMe 3 in MeOH. SANS data analysis for P3HT- $\boldsymbol{b}$ CPEs. AFM images of P3HT-b-CPEs drop-cast from $\mathrm{MeOH}, \mathrm{H}_{2} \mathrm{O}$ or $\mathrm{H}_{2} \mathrm{O} /$ mixture. See DOI: 10.1039/b000000x/ 
1 Conjugated polyelectrolytes. Fundamentals and Applications (Eds. B. Liu and G. C. Bazan) 2013, Wiley-VCH, Weinheim.

2 a) W. Lee, J. H. Seo and H. Y. Woo, Polymer, 2013, 54, 5104; b) A. Duarte, K.-Y. Pu, B. Liu and G. C. Bazan, Chem. Mater., 2011, 23 , 501; c) H. Jiang, P. Taranekar, J. R. Reynolds and K. S. Schanze, Angew. Chem. Int. Ed., 2009, 48, 4300; d) C. Zhu, L. Liu, Q. Yang, F. Lv and Shu Wang, Chem. Rev., 2012, 112, 4687; e) G. Feng, J. Liang and B. Liu, Macromol. Rapid Commun., 2013, 34, 705; f) G. Feng, D. Ding and B. Liu, Nanoscale, 2012, 4, 6150.

3 a) T. Q. Nguyen, R. C. Kwong, M. E. Thompson and B. J. Schwartz, Appl. Phys. Lett., 2000, 76, 2454; b) C. L. Chochos and S. A Choulis, Prog. Polym. Sci., 2011, 36, 1326; c) K. Vandewal, S. Himmelberger and A. Salleo, Macromolecules, 2013, 46, 6379.

4 R. C. Evans, J. Mater. Chem. C, 2013, 1, 4190 and references therein.

5 a) M. Knaapila, R. C. Evans, V. M. Garamus, L. Almasy, N. K. Szekely, A. Gutacker, U. Scherf and H. D. Burrows, Langmuir, 2010, 26, 15634; b) M. Knaapila, R. C. Evans, A. Gutacker, V. M. Garamus, N. K. Szekely, U. Scherf and H. D. Burrows, Soft Matter, 2011, 7, 6863; c) R. C. Evans, M. Knaapila, N. Willis-Fox, M. Kraft, A. Terry, H. D. Burrows and U. Scherf, Langmuir 2012, 28, 12348; d) Y.-M. Chang, R. Zhu, E. Richard, C.-C. Chen, G. Li and Y. Yang, Adv. Funct. Mater., 22, 3284; e) M. E. H. Heeley, J. K. Gallaher, T. L. Nguyen, H. Y. Woo and J. M. Hodgkiss, Chem. Commun. 2013, 49, 4235 .

6 H. Jiang, X. Zhao and K. S. Schanze, Langmuir, 2006, 22, 5541.

7 a) H. D. Burrows, S. M. Fonseca, F. B. Dias, J. Seila de Melo, A. P. Monckman, U. Scherf and S. Pradhan, Adv. Mater., 2009, 21, 1155. b) J. E. Houston, A. R. Patterson, A. C. Jayasundera, W. Schmitt and R. C. Evans, Chem. Commun., 2014, DOI:10.1039/C3CC47552B.

8 J. Rubio-Magnieto, A. Thomas, S. Richeter, A. Mehdi, P. Dubois, R. Lazzaroni, S. Clément and M. Surin, Chem. Commun., 2013, 49, 5483 .

9 a) U. Scherf, A. Gutacker and N. Koenen, Acc. Chem. Res., 2008, 41, 1086; b) P.-T. Wu, G. Ren, F. S. Kim, C. Li, R. Mezzenga and S. A. Jenekhe, J. Polym. Sci., Part A: Polym. Chem., 2010, 48, 614; c) J. Kim, A. Siva, I. Y. Song and T. Park, Polymer, 2011, 52, 3704; d) Y. Lin, J. A. Lim, Q. Wei, S. B. Mannsfeld, A. L. Briseno and J. J. Watkins, Chem. Mater., 2012, 24, 622; e) A. Yassar, L. Miozzo, R. Gironda and G. Horowitz, Prog. Polym. Sci., 2013, 38, 791; f) M. J. Robb, S.-Y. Ku and C. J. Hawker, Adv. Mater., 2013, 25, 5686.

10 a) Y. Zhang, T. Keisuke, H. Kouske and H. Kazuhito, J. Am. Chem. Soc., 2008, 130, 7812; b) Y. Zhang, K. Tajima and K. Hashimoto, Macromolecules, 2009, 42, 7008.

11 a) D. M. Vriezema, M. C. Aragonés, J. A. A. W. Elemans, J. J. L. M. Cornelissen, A. E Rowan and R. J. M. Nolte, Chem. Rev., 2005, 105, 1445; b) A. Kros, W. Jesse, G. A. Metselaar and J. J. L. M. Cornelissen, Angew. Chem. Int. Ed. 2005, 44, 4349; c) R. Verduzco, J. Botiz, D. L. Pickel, S. M. Kilbey, K. Hong, E. Dimasi and S. B. Darling, Macromolecules, 2011, 44, 530.

12 a) G. L. Tu, H. B. Li, M. Forster, R. Heiderhoff, L. J. Balk, R. Sigel, U. Scherf, Small, 2007, 3, 1001; b) A. Gutacker, S. Adamczyk, A. Helfer, L. E. Garner, R. C. Evans, S. M. Fonseca, M. Knaapila, G. C. Bazan, H. D. Burrows and U. Scherf, J. Mater. Chem. 2010, 20, 1423; c) A. Gutacker, N. Koenen, U. Scherf, S. Adamczyk, J. Pina, S. M. Fonseca, A. J. M. Valente, R. C. Evans, J. Seixas de Melo, H. D.
Burrows and M. Knaapila, Polymer, 2010, 51, 1898; d) M. Knaapila, R. C. Evans, A. Gutacker, V. M. Garamus, M. Torkkeli, S. Adamczyk, M. Forster, U. Scherf and H. D. Burrows, Langmuir, 2010, 26, 5056

13 a) U. Scherf, R. C. Evans, A. Gutacker and G. C. Bazan in Conjugated polyelectrolytes. Fundamentals and Applications (Eds. B. Liu and G. C. Bazan) 2013, Wiley-VCH, Weinheim, pp 65-90; b) A. Gutacker, C.-Y. Lin, L. Ying, T.-Q. Nguyen, U. Scherf, and G. C. Bazan, Macromolecules, 2012, 45, 4441.

14 T. Ghoos, J. Brassinne, C.-A. Fustin, J.-F. Gohy, M. Defour, N. Van den Brande, B. Van Mele, L. Lutsen, D. J. Vanderzande and W. Maes, Polymer, 2013, 54, 6293.

15 a) R. D. McCullough, Adv. Mater., 1998, 10, 93; b) K. Faied, M. Frechette, M. Ranger, L. Mazerolle, I. Levesque, M. Leclerc, T.-A. Chen and R. D. Rieke, Chem. Mater., 1995, 7, 1390.

16 M. Trung Dang, L. Hirsch, G. Wantz and J. D. Wuest, Chem. Rev., 2013, 113, 3734.

17 a) K. Yao, L. Chen, Y. Chen, F. Li and P. Wang, J. Mater. Chem., 2011, 21, 13780; b) J. H. Seo, A. Gutacker, Y. M. Sun, H. B. Wu, F. Huang, Y. Cao, U. Scherf, A. J. Heeger and G. C. Bazan, J. Am. Chem. Soc., 2011, 133, 8416; c) Y. M. Chang, R. Zhu, E. Richard, C.-C. Chen, G. Li and Y. Yang, Adv. Funct. Mater., 2012, 22, 3284.

18 a) J. Kesters, T. Ghoos, H. Penxten, J. Drijkoningen, T. Vangerven, D. M. Lyons, B. Verreet, T. Aernouts, L. Lutsen, D. Vanderzande, J. Manca and W. Maes, Adv. Energy Mater., 2013, 3, 1180; b) S. Khiev, L. Derue, G. Ayenew, H. Medlej, R. Brown, L. Rubatat, R. C. Hiorns, G. Wantz and C. Dagron-Lartigau, Polym. Chem., 2013, 4, 4145.

19 a) I. Osaka and R. D. McCullough, Acc. Chem. Res., 2008, 41, 1202; b) K. Okamoto and C. K. Luscombe, Polym. Chem. 2011, 2, 2424; c) A. Kiriy, V. Senkovskyy and M. Sommer, Macromol. Rapid Commun., 2011, 32, 1503.

20 a)A. E. Javier, S. R. Varshney and R. D. McCullough, Macromolecules, 2010, 43, 3233; b) A; Sui, X. Shi, S. Wu, H. Tian, Y. Geng and F. Wang, Macromolecules, 2012, 45, 5436.

21 M. C. Stefan, A. E. Javier, I. Osaka and R. D. McCullough, Macromolecules, 2008, 42, 30.

22 M. A. Green, K. Emery, Y. Hishikawa and W. Warta, Prog. Photovolt. Res. Appl., 2009, 17, 85.

23 a) S. Clément, F. Meyer, J. De Winter, O. Coulembier, C. M. L. Vande Velde, M. Zeller, P. Gerbaux, J.-Y. Balandier, S. Sergeyev, R. Lazzaroni, Y. Geerts and P. Dubois, J. Org. Chem., 2010, 75, 1561; b) M. C. Stefan, M. P. Bhatt, P. Sista and H. D. Magurudeniya, Polym. Chem., 2012, 3, 1693; c) R. J. Ono, S. Kang and C. W. Bielawski, Macromolecules, 2012, 45, 2321; d) Y.-H. Lin, Yen-K. A. Smith, C. N. Kempf and R. Verduzco, Polym. Chem., 2013, 4, 229.

24 S. Clément, A. Tizit, S. Desbief, A. Mehdi, J. De Winter, P. Gerbaux, R. Lazzaroni and B. Boury, J. Mater. Chem., 2011, 21, 2733.

25 J. De Winter, G. Deshayes, F. Boon, O. Coulembier, P. Dubois, P. Gerbaux, J. Mass Spectrom., 2011, 46, 237.

26 R. L. Danley, P. A. Caulfield and S. R. Aubuchon, Am. Lab., 2008, 40, 9 .

27 S. Wouters, F. Demir, L. Beenaerts and G. Van Assche, Thermochim. Acta, 2012, 530, 64.

28 P. G. Seybold and M. Gouterman, J. Mol. Spectrosc., 1969, 31, 1. 
29 International Standard ISO22412 Particle Size Analysis - Dynamic Light Scattering, International Organisation for Standardisation (ISO) 2008.

30 R.K. Heenan, S.E. Rogers, D. Turner, A. E. Terry, J. Treadgold and S. M. King, Neutron News, 2011, 22, 19.

31 http://www.mantidproject.org

32 G. D. Wignall and F. S. Bates, J. Appl. Crystallogr., 1987, 20, 28.

33 I. Livsey, J. Chem. Soc. Faraday Trans. 2, 1987, 83, 1445.

34 a) L. Zhai, R. L. Pilston, K. L. Zaiger, K. K. Stokes and R. D. McCullough, Macromolecules, 2003, 36, 61; b) S. Miyanishi, K. Tajima and K. Hashimoto, Macromolecules, 2009, 42, 1610.

35 R. Miyakoshi, A. Yokoyama and T. Yokozawa, Macromol. Rapid Commun., 2004, 25, 1663.

36 J. Liu, R. S. Loewe and R. D. McCullough, Macromolecules, 1999, 32, 5777.

37 R. Puffr and J. Sebenda, J. Polym. Sci., 1967, 16, 79

38 a) R. D. McCullough, S. Tristram-Nagle, S. P. Williams, R. D. Lowe and M. Jayaraman, J. Am. Chem. Soc., 1993, 115, 4910; b) W. D. Oosterbaan, V. Vrindts, S. Berson, S. Guillerez, O. Douhéret, B. Ruttens, J. D'Haen, P. Adriaensens, J. Manca, L. Lutsen and D. Vanderzande, J. Mater. Chem., 2009, 19, 5424.

39 M. Sundberg, O. Inganäs, S. Stafström, G. Gustafsson, B. Sjögren, Solid State Comm., 1989, 71, 435.

40 a) C. Tan, M. R. Pinto and K. S. Schanze, Chem. Commun., 2002, 446; b) S. Wang and G. C. Bazan, Chem. Commun., 2004, 2508; c) H. D. Burrows, S. M. Fonseca, C. L. Silva, A. A. C. C. Pais; M. J. Tapia, S. Pradhan and U. Scherf, Phys. Chem. Chem. Phys., 2008, 10, 4420.

41 P. W. Schmidt, Some Fundamental Concepts and Techniques Useful in Small-Angle Scattering Studies of Disordered Solids. In Modern Aspects of Small-Angle Scattering; H. Brumberger,, Ed.; Kluwer Academic Publishers: Dordrecht, The Netherlands, 1995; pp 1-56.

42 CRC Handbook of Chemistry and Physics; D. W. H. Rankin, Eds.; Taylor \& Francis: New York, 2009. 